\title{
Microbial Engraftment and Efficacy of Fecal Microbiota Transplant for Clostridium Difficile in Patients With and Without Inflammatory Bowel Disease
}

\author{
Robert P. Hirten, MD, ${ }^{*, \#}$ Ari Grinspan, MD, ${ }^{*, *}$ Shih-Chen Fu,PhD, ${ }^{+, \#}$ Yuying Luo, MD, Mayte Suarez-Farinas, PhD, \\ John Rowland, PhD, ${ }^{\ddagger}$ Eduardo J. Contijoch, PhD, ${ }^{+}$Ilaria Mogno, PhD, ${ }^{+}$Nancy Yang, MD, ${ }^{*}$ Tramy Luong, BS, \\ Philippe R. Labrias, BS, ${ }^{+}$Inga Peter, PhD, ${ }^{+}$Judy H. Cho, MD, ${ }^{+}$Bruce E. Sands, MD, ${ }^{*}$ Jean Frederic Colombel, MD, ${ }^{*}$ \\ Jeremiah J. Faith, $P h D^{t, \S}$ and Jose C. Clemente, $P h D^{t, \S}$
}

Background: Recurrent and severe Clostridium difficile infections (CDI) are treated with fecal microbiota transplant (FMT). Uncertainty exists regarding FMT effectiveness for CDI with underlying inflammatory bowel disease (IBD) and regarding its effects on disease activity and effectiveness in transferring the donor microbiota to patients with and without IBD.

Methods: Subjects with and without IBD who underwent FMT for recurrent or severe CDI between 2013 and 2016 at The Mount Sinai Hospital were followed for up to 6 months. The primary outcome was CDI recurrence 6 months after FMT. Secondary outcomes were (1) CDI recurrence 2 months after FMT; (2) frequency of IBD flare after FMT; (3) microbiota engraftment after FMT; (and 4) predictors of CDI recurrence.

Results: One hundred thirty-four patients, 46 with IBD, were treated with FMT. Follow-up was available in 83 and 118 patients at 6 and 2 months, respectively. There was no difference in recurrence in patients with and without IBD at 6 months $(38.7 \%$ vs $36.5 \% ; P>0.99)$ and 2 months $(22.5 \%$ vs $17.9 \%$; $P=0.63)$. Proton pump inhibitor use, severe CDI, and comorbid conditions were predictors of recurrence. Pre-FMT microbiota was not predictive of CDI recurrence. Subjects with active disease requiring medication escalation had reduced engraftment, with no difference in engraftment based on CDI recurrence or IBD endoscopic severity at FMT.

Conclusions: Inflammatory bowel disease did not affect CDI recurrence rates 6 months after FMT. Pre-FMT microbiota was not predictive of recurrence, and microbial engraftment was impacted in those requiring IBD treatment escalation, though not by CDI recurrence or IBD disease severity.

Key Words: Clostridium difficile, microbiome, inflammatory bowel disease, fecal microbiota transplant 2018

Received for publications August 27, 2018; Editorial Decision December 19,

From the ${ }^{*}$ The Dr. Henry D. Janowitz Division of Gastroenterology, Icahn School of Medicine at Mount Sinai, New York, NY, USA; Icahn Institute for Genomics \& Multiscale Biology, Department of Genetics and Genomic Sciences, Icahn School of Medicine at Mount Sinai, New York NY, USA; ${ }^{\star}$ Center for Biostatistics, Department of Population Health Science and Policy, Icahn School of Medicine at Mount Sinai, New York, NY, USA; \$Precision Immunology Institute, Icahn School of Medicine at Mount Sinai, New York NY, USA

${ }^{\#}$ Robert P Hirten, Ari Grinspan, Shih-Chen Fu should be considered joint first authors.

Author Contribution: RPH, AG, SCF, and JCC contributed to the conception and design. RPH, AG, SCF, JCC, LY, MSF, JR, EJC, IM, NY, TL, PL, and JJF contributed to the generation, collection, assembly, analysis, and/or interpretation of the data. RPH, AG, SCF, LY, MSF, JR, EJC, IM, NY, TL, PRL, IP, JHC, BES, JFC, JJF, and JCC contributed to the drafting or revision of the manuscript. All authors read, reviewed, and approved the final version of the manuscript and the authorship list.

Conflicts of Interest:RPH served as a consultant, advisory board member or speaker for Janssen and Takeda. JFC has served as consultant, advisory board member or speaker for AbbVie, Amgen, Boehringer-Ingelheim, Celgene Corporation, Celltrion, Enterome, Ferring, Genentech, Janssen and Janssen, Lilly, Medimmune, Merck \& Co., Pfizer, PPM Services, Protagonist, Second Genome, Seres, Shire, Takeda, Theradiag, Theravance Biopharma; he has also served as speaker for AbbVie, Ferring, Pfizer, Takeda, Shire and has stock options with Intestinal Biotech Development, Genfit; he has received research grants from AbbVie, Takeda, and Janssen and Janssen. JJF has served as consultant, advisory board member or speaker for Vedanta Biosciences and Janssen; he has received research grants from Janssen. BES has received consulting fees and research grants from AbbVie, Pfizer, Amgen, Bristol-Myers Squibb, Celgene, Janssen, and Takeda and has received consulting fees from Boehringer Ingelheim,
Akros Pharma, Arena Pharmaceuticals, Forward Pharma, Immune Pharmaceuticals, Lilly, Synergy Pharmaceuticals, Theravance, Receptos, TiGenix, TopiVert Pharma, MedImmune, Vedanta Biosciences, Allergan, UCB Pharma, EnGene, Target PharmaSolutions, Lycer, Lyndra, Vivelix Pharmaceuticals, Oppilan Pharma, and Gilead. JHC serves as a consultant for Pfizer and has a sponsored research agreement with Goldfinch Bio. All other authors declare no conflict of interest.

Address correspondence to: Jose C. Clemente, 1470 Madison Avenue, New York NY, USA 10029. E-mail: jose.clemente@mssm.edu.

Abbreviations: Anti-TNF, anti-tumor necrosis factor; AZA, azathioprine; BMI, body mass index; CD, Crohn's disease; CDI, clostridium difficile infection; CDIR, clostridium difficile infection relapse; CRP, c-reactive protein; ESR, erythrocyte sedimentation rate; FMT, fecal microbiota transplant; GERD, gastroesophageal reflux disease; HBI, Harvey Bradshaw Index; IBD, inflammatory bowel disease; Non-IBD, non-inflammatory bowel disease group; $\mathrm{IBD}^{\mathrm{e}}$, inflammatory bowel disease medication escalation; IBD ${ }^{\mathrm{s}}$, inflammatory bowel disease no medication escalation; $\mathbf{J}$ tube, jejunal tube; LSM, least-squares means; MP, mercaptopurine; MTX, methotrexate; OTU, operational taxonomic unit; PCoA, principal coordinate analysis; PEG, percutaneous endoscopic gastrostomy; PPI, proton pump inhibitor; PUD, peptic ulcer disease; SD, standard deviation; SES-CD, simple endoscopic score for Crohn's disease; UC, ulcerative colitis; WBC, white blood cell count

Supported in part by the SUCCESS (Sinai Ulcerative Colitis Clinical, Experimental and System Studies) grant from the Bacchetta Foundation (SCF, IP, JHC, JFC, JJF, JCC), the Crohn's and Colitis Foundation of America grant \#362048 (JJF, JCC), the George Waechter Memorial Foundation grant (AG), and NIH/ NIDDK R01 DK114038 (JCC).

(C) 2019 Crohn's \& Colitis Foundation. Published by Oxford University Press. All rights reserved. For permissions, please e-mail: journals.permissions@oup.com. doi: 10.1093/ibd/izy398 Published online 10 March 2019 


\section{INTRODUCTION}

Clostridium difficile infection (CDI) is one of the most common health-care associated infections and is associated with significant morbidity and mortality. ${ }^{1}$ After initial antibiotic therapy, $10 \%-20 \%$ of patients will experience a recurrence, and up to $65 \%$ will recur after subsequent episodes. ${ }^{2,3}$ Generally, the first recurrence is treated with the same antibiotic regimen used for the initial infection, whereas fidaxomicin, a prolonged vancomycin course and fecal microbiota transplant (FMT), are used for the second and third recurrences. ${ }^{4}$

Clostridium difficile infection has been associated with alterations of the intestinal microbiome, generally reducing bacterial diversity and the abundance of Bacteroidetes and Firmicutes phyla. ${ }^{5}$ Fecal microbiota transplant effectively treats recurrent CDI in approximately $90 \%$ of patients, though its exact mechanism remains unclear. Although FMT is currently used in clinical practice to treat CDI in patients with IBD, studies have demonstrated variable efficacy in this population. ${ }^{6,7}$ There are concerns regarding the use of FMT in patients with underlying IBD due to the frequent use of concomitant immunosuppressive agents and the possibility of worsening IBD activity. Several studies found a worsening of IBD activity in up to $23 \%$ of patients post-FMT. ${ }^{6-9}$ Furthermore, it is unknown whether microbiome engraftment is lower in patients with concomitant IBD compared with those with CDI only, which could result in increased recurrence rates.

Given these questions and the lack of published data on the long-term efficacy of FMT, with most studies focused on recurrence rates within 1 to 3 months of the transplant, the goal of this study was to determine FMT's long-term effectiveness in the treatment of CDI and the predictors of post-FMT recurrence in patients with and without IBD. Evaluation of the microbiome was also performed in a subset of patients to assess the impact of IBD on engraftment and its subsequent risk of relapse.

\section{MATERIALS AND METHODS}

\section{Study Design}

This is a longitudinal, retrospective cohort study including all patients 18 years or older with and without IBD who underwent FMT for recurrent or severe CDI between 2013 and 2016 at The Mount Sinai Hospital (New York, USA). Eligibility criteria for FMT at our institution included recurrent CDIs characterized as (1) at least 3 episodes of mild to moderate CDI and failure of a 6- to 8-week taper with vancomycin and (2) at least 2 episodes of severe CDI resulting in hospitalizations and associated with significant morbidity. Eligibility also included severe CDIs characterized as (1) persistent moderate to severe CDI not responding to standard therapy (vancomycin) for at least 1 week and (2) severe (including fulminant) CDI with no response to standard therapy after 48 hours.
All patients received standard dose vancomycin before FMT. The mode of FMT delivery was determined by the treating physician's clinical judgement and was performed preferentially via colonoscopy or flexible sigmoidoscopy, with patients taking the colonoscopy bowel preparation as a split dose starting the prior evening. Colonoscopy was performed to the terminal ileum if possible, with the fecal suspension instilled in the most proximally reached portion of the intestine. Fecal microbiota transplant done via the upper gastrointestinal tract was performed via push enteroscopy, percutaneous endoscopic gastrostomy (PEG) tube, or jejunal (J) tube. Push enteroscopy was performed to the proximal jejunum where the fecal suspension was instilled. When performed through the PEG tube or $\mathrm{J}$ tube, the fecal suspension was instilled into the stomach or jejunum and flushed with $40 \mathrm{cc}$ of nonbacteriostatic normal saline. Stool for transplant was obtained from either healthy donors screened for relevant communicable diseases (fresh), CIPAC Therapeutics (frozen), or OpenBiome (frozen).

Baseline demographic data and the medical and surgical history for all patients were collected. Inflammatory bowel disease activity at the time of FMT and at 2 and 6 months after transplant was recorded and characterized utilizing clinical disease activity scores (Harvey-Bradshaw index [HBI] for CD and the partial Mayo Score for UC). Endoscopic IBD severity was captured at the time of FMT utilizing endoscopic grading systems (Simple Endoscopic Score for Crohn's Disease [SES-CD] and The Mayo endoscopic subscore). The HBI was classified as $<5$ (remission), 5 to 7 (mild disease), 8 to 16 (moderate disease), and $>16$ (severe disease). The partial Mayo score was classified as 0 to 1 (remission), 2 to 4 (mild disease), 5 to 6 (moderate disease), and 7 to 9 (severe disease). An SES-CD score was classified as 0 to 2 (remission), 3 to 6 (mild activity), 7 to 15 (moderate activity), and $>15$ (severe activity). An endoscopic Mayo subscore was classified as 0 (remission), 1 (mild activity), 2 (moderate activity), and 3 (severe activity). Inflammatory bowel disease-related medications were captured before FMT and longitudinally in the subset of patients in whom the microbiome was analyzed to assess for therapeutic escalation. Therapeutic escalation was defined as the need to initiate new IBD treatment including corticosteroids and biologic medication or the need to change the current medication. The severity of the CDI was defined by the 2013 American College of Gastroenterology guidelines. ${ }^{3}$

The primary outcome was late CDI recurrence at 6 months after initial FMT in patients with and without IBD and in the cohort as a whole. The secondary outcomes were (1) early CDI recurrence at 2 months after initial FMT; (2) frequency of IBD flare at 2 and 6 months after initial FMT; (3) microbiome engraftment after FMT; and (4) predictors of CDI recurrence after initial FMT. Successful FMT was defined as a resolution of diarrhea within 8 weeks of the transplant and no need for re-initiation of therapy. Recurrence of CDI is defined as a recurrence of diarrhea and laboratory confirmation of $C$. difficile in the stool. Clostridium difficile infection was 
diagnosed utilizing a 2-step algorithm using an initial enzyme immunoassay for glutamate dehydrogenase antigen and toxin $\mathrm{A}$ or $\mathrm{B}$, with a confirmatory polymerase chain reaction (PCR) obtained for discordant results. An IBD flare was diagnosed by the treating physician based upon clinical symptoms and the need for IBD-related medication escalation or change.

\section{Microbiome Data Generation and Analysis}

Subjects were approached sequentially at the time of FMT to take part in a longitudinal analysis of their microbiome. The first 29 subjects who were scheduled to receive an FMT from a fresh donor $(n=19)$ and consented to participate provided samples before FMT. Out of those, 18 subjects with $(n=9)$ and without $(n=9)$ IBD had their microbiome analyzed before FMT and up to 12 months after microbiota transplantation. Samples for microbiome analysis were collected the day before FMT, at the time of FMT, within 48 hours after transplant, 1 week after FMT, 4 weeks after FMT, 8 weeks after FMT, 6 months after FMT, and 12 months after FMT. These 18 subjects received fresh FMT from 1 of 11 out of the 19 initial healthy donors who also had their stool analyzed. Fecal microbiota was analyzed utilizing 16S rRNA sequencing as described previously. ${ }^{10}$ Briefly, human fecal samples were collected fresh and stored at $-80^{\circ} \mathrm{C}$ before processing. After suspension in extraction buffer, samples were mechanically lysed, centrifuged, and DNA extracted. The V4 variable region of the $16 \mathrm{~S}$ rRNA gene was amplified by PCR using indexed primers as previously described. ${ }^{11}$ Uniquely indexed 16S rDNA V4 amplicons were pooled and purified, and the pooled samples were sequenced with an Illumina MiSeq (paired-end $250 \mathrm{bp}$ ). Paired end reads were joined into a single DNA sequencing using the FLASH algorithm. ${ }^{12}$ We obtained a total of 7,263,850 reads (average 59,539 $\pm 34,744$ reads/sample) after demultiplexing and quality filtering as previously described. ${ }^{10}$ Data were then clustered into Operational Taxonomic Units (OTUs) using a closed-reference OTU picking algorithm ${ }^{13}$ against Greengenes v13-8, ${ }^{14}$ resulting in a total of 6,053 OTUs. Alpha diversity was estimated using Faith's phylogenetic diversity, ${ }_{15}^{15}$ and beta diversity was estimated using unweighted UniFrac, ${ }^{16}$ as both implemented in QIIME v1.9.1. ${ }^{17}$ For simplicity, we will refer to "alpha diversity" and "beta diversity" hereafter. Microbiome engraftment was estimated using unweighted UniFrac distance from patient to donor microbiome. High engraftment is thus represented by smaller distances (perfect engraftment would have distance 0 , as there are no differences between the microbiome of donor and recipient), whereas low engraftment has larger distances (if donor and recipient microbiome have absolutely no overlap, the distance would be 1). Metagenomic functions were predicted using PICRUSt (Phylogenetic Investigation of Communities by Reconstruction of Unobserved States), and differential analysis of pathways was performed using STAMP (Statistical Analysis of Metagenomic Profiles). ${ }^{18,19}$

\section{Statistical Analysis}

Baseline comparisons of categorical data in subjects with and without IBD were conducted using the Fischer exact test and $\chi^{2}$ test. The $t$ test was used for continuous data. Recurrence rates of CDI in IBD and non-IBD groups were presented with 95\% confidence intervals (Cis), computed using the results of the proportion test within each group, and compared between the groups at 2-month and 6-month endpoints using the Fischer exact test. The time to first CDI recurrence was compared using the Log-rank test and presented as a Kaplan-Meier curve. Changes in continuous outcomes over time were compared between IBD and non-IBD groups using linear mixed-effects models. To evaluate clinical variables as predictors of CDI recurrence, a 2-step strategy was set up where the most robust predictors were identified by combining multiple imputations and regularized regression techniques and fit to a multivariable regression model.

The $\mathrm{R}$ version 3.4.1 was utilized unless otherwise noted. The log-rank test, presented as a Kaplan-Meier curve, was done utilizing R's packages survival (version 3.1-131) and survminer (version 0.4.0). Changes in continuous outcomes over time were done using linear mixed-effects models using R's package NLME (version 3.1-131) and lsmeans (version 2.27-2).

\section{ETHICAL CONSIDERATIONS}

This study was approved by the Institutional Review Board of the Icahn School of Medicine at The Mount Sinai Hospital, and all subjects provided their informed consent before inclusion.

\section{RESULTS}

\section{Patient Population}

A total of 134 patients with CDI were treated with FMT, 46 of whom had underlying IBD (Table 1). Among IBD patients, 27 patients had ulcerative colitis (UC), 18 patients had Crohn's disease (CD), and 1 patient had indeterminate colitis (Supplementary Table S1). Sixty-four percent of the cohort were women, and the average age was 53 years. The cohort with IBD was significantly younger than the non-IBD cohort (mean age 38.8 vs 60.3 years; $P<0.001$ ). The indication for FMT was for recurrent CDI in 89 patients and severe CDI in 44 patients. This did not differ between the IBD and non-IBD groups $(P=0.39)$. Of the FMTs performed, $21.6 \%$ were done in the inpatient setting and $78.4 \%$ in the outpatient setting, with fresh and frozen stool used in $51.5 \%$ and $48.5 \%$ of FMTs, respectively. There were $51.5 \%$ of patients hospitalized within the 90 days before FMT, which was significantly more frequent in non-IBD patients compared with IBD patients $(58 \%$ vs $39.1 \% ; P=0.04)$, as was the percentage of patients requiring a past hospitalization for CDI $(55.7 \%$ vs $34.8 \% ; P=0.02)$. At the time of fecal transplant, $91.3 \%$ of IBD patients were receiving an immunosuppressive agent. At FMT, 37 (82\%) patients with IBD had evidence of endoscopic disease activity. Among 
TABLE 1. Patient Characteristics of the Cohort

\begin{tabular}{|c|c|c|c|}
\hline & IBD $(\%)(n=46)$ & Non-IBD $(\%)(n=88)$ & $P$ \\
\hline Age, mean \pm SD & $38.8 \pm 20.5$ & $60.3 \pm 18.9$ & $<0.001$ \\
\hline Female sex & $25(54.3)$ & $61(69.3)$ & 0.09 \\
\hline $\mathrm{BMI}$ at $\mathrm{FMT}$, mean $\pm \mathrm{SD}$ & $24.6 \pm 6.7$ & $25.8 \pm 8.2$ & 0.37 \\
\hline Hospitalization 90 days before FMT & $18(39.1)$ & $51(58)$ & 0.04 \\
\hline Proton Pump Inhibitor & $15(32.6)$ & $35(40.2)$ & 0.39 \\
\hline Number of Prior CDI, mean \pm SD & $3.5 \pm 1.5$ & $3.5 \pm 2.0$ & 0.88 \\
\hline FMT Indication & & & 0.39 \\
\hline Refractory & $13(28.3)$ & $31(35.6)$ & \\
\hline Recurrent & $33(71.7)$ & $56(64.4)$ & \\
\hline Donor Type & & & 0.53 \\
\hline Fresh & $22(47.8)$ & $45(53.6)$ & \\
\hline Frozen & $24(52.2)$ & $39(46.4)$ & \\
\hline Location of Transplant & & & 0.08 \\
\hline Inpatient & $6(13)$ & $23(26.1)$ & \\
\hline Outpatient & $40(87.0)$ & $65(73.9)$ & \\
\hline \multicolumn{4}{|l|}{ Laboratory Findings at FMT } \\
\hline $\mathrm{CRP}$, mean $\pm \mathrm{SD}$ & $13.6 \pm 20.9$ & $33.4 \pm 75.3$ & 0.21 \\
\hline $\mathrm{ESR}$, mean $\pm \mathrm{SD}$ & $33 \pm 29.8$ & $37.7 \pm 38.5$ & 0.60 \\
\hline Albumin, mean $\pm \mathrm{SD}$ & $3.6 \pm 0.7$ & $3.3 \pm 0.9$ & 0.19 \\
\hline $\mathrm{WBC}$, mean $\pm \mathrm{SD}$ & $8.6 \pm 3.7$ & $11.3 \pm 11.6$ & 0.09 \\
\hline Hemoglobin, mean \pm SD & $11.6 \pm 1.6$ & $11.3 \pm 2.1$ & 0.41 \\
\hline \multicolumn{4}{|l|}{ Comorbid Conditions } \\
\hline Anxiety/Depression & $10(21.7)$ & $18(20.5)$ & 0.86 \\
\hline GERD or PUD & $5(10.9)$ & $20(22.7)$ & 0.09 \\
\hline Hypertension & $6(13)$ & $38(43.2)$ & $<0.001$ \\
\hline Psoriasis & $3(6.5)$ & $3(3.4)$ & 0.41 \\
\hline Cardiovascular disease & $8(17.4)$ & $35(40.7)$ & 0.006 \\
\hline Diet Intolerance & $4(8.7)$ & $1(1.1)$ & 0.05 \\
\hline Diabetes Mellitus & $2(4.3)$ & $18(20.5)$ & 0.01 \\
\hline Irritable Bowel Syndrome & $1(2.2)$ & $9(10.2)$ & 0.16 \\
\hline Kidney Disease & $2(4.3)$ & $14(15.9)$ & 0.05 \\
\hline Autoimmune Disease & $3(6.5)$ & $12(14)$ & 0.20 \\
\hline Liver Disease & $2(4.3)$ & $15(17.6)$ & 0.03 \\
\hline Antibiotic Exposure & $24(57.1)$ & $70(83.3)$ & 0.001 \\
\hline Previous CDI Hospitalization & $16(34.8)$ & $49(55.7)$ & 0.02 \\
\hline CDI Severity & & & 0.22 \\
\hline Mild & $26(57.8)$ & $47(53.4)$ & \\
\hline Moderate & $13(28.9)$ & $22(25.0)$ & \\
\hline Severe & $6(13.3)$ & $19(21.6)$ & \\
\hline Prior Metronidazole Courses, mean \pm SD & $1.0 \pm 0.8$ & $1.2 \pm 0.8$ & 0.23 \\
\hline Prior Vancomycin Courses, mean \pm SD & $2.6 \pm 1.3$ & $2.3 \pm 1.5$ & 0.15 \\
\hline Prior Fidaxomicin Courses, mean $\pm \mathrm{SD}$ & $0.2 \pm 0.5$ & $0.3 \pm 0.7$ & 0.16 \\
\hline Colon Polyps & $7(15.2)$ & $13(14.9)$ & 0.97 \\
\hline Colonic Strictures & $4(8.7)$ & $3(3.4)$ & 0.23 \\
\hline Hemorrhoids & $3(6.5)$ & $7(8.0)$ & $>0.99$ \\
\hline Diverticulosis & $2(4.3)$ & $35(40.2)$ & $<0.001$ \\
\hline
\end{tabular}

BMI, body mass index; CRP, c-reactive protein; ESR, erythrocyte sedimentation rate; WBC, white blood cell count; GERD, gastroesophageal reflux disease; PUD, peptic ulcer disease; $\mathrm{SD}$, standard deviation. 
cohort patients, $18.8 \%$ had a severe CDI, with severity not differing between those with or without IBD $(P=0.22)$.

We noted differences in comorbid conditions between the 2 groups, with significantly fewer patients with IBD having hypertension $(P<0.001)$, cardiovascular disease $(P=0.006)$, diabetes mellitus $(P=0.01)$, and liver disease $(P=0.03)$. Additionally, patients with IBD were less likely than patients without IBD to have diverticulosis seen on colonoscopy at the time of FMT $(4.3 \%$ vs $40.2 \% ; P<0.001)$.

\section{Outcomes and Predictors of Failure}

Our primary outcome was late CDI recurrence 6 months after FMT. Thirty-one out of $83(37.3 \%)$ patients with follow-up at 6 months suffered from recurrent CDI after the initial FMT. Subjects with IBD did not have a higher rate of CDI recurrence at 6 months $(38.7 \%$ vs $36.5 \% ; P>0.99)$ compared with the non-IBD group (Fig. 1), and there was no difference between the groups in time to first recurrence $(P=0.46)$. At 6 months, 21 out of 76 patients $(27.6 \%)$ for whom data was available required repeat FMT, which was not significantly higher in the group with IBD compared with those without IBD $(25.0 \%$ vs $29.2 \% ; P=0.70)$. There were no serious adverse events noted secondary to FMT during the 6-month follow-up period, and no difference in colectomy rate in the IBD and nonIBD groups were observed ( $12.9 \%$ vs $9.5 \% ; P=0.72)$.

Short-term recurrent CDI occurred in 23 out of $118(19.5 \%)$ patients with follow-up at 2 months from initial FMT. Subjects with IBD did not have a higher rate of recurrence at 2 months compared with those without IBD $(22.5 \%$ vs $17.9 \% ; P=0.63)$. Eighteen of the 107 subjects $(16.8 \%)$ with available follow-up data required repeat FMT by 2 months of follow-up, which was not significantly higher in the group with IBD compared with those without IBD (17.2\% vs $16.7 \%$; $P>0.99)$.

Univariate analysis did not reveal any factors associated with the risk of CDI recurrence at 2 months. Recurrence of CDI at 6 months was associated with the use of proton pump inhibitors $(P=0.01)$, FMT performed as an inpatient $(P=0.02)$, and a lower hemoglobin $(P=0.02)$ (Table 2). At 6 months and 2 months, respectively, IBD type $(P=0.13 ; P=0.71)$, immunosuppression at FMT $(P>0.99 ; P=0.55)$, and IBD severity at FMT $(P=0.63 ; P>0.99)$ were not predictors of CDI recurrence. Based on the final logistic regression model, proton pump inhibitor use $(P=0.045)$, severe CDI at the time of FMT $(P=0.005)$, and hypertension $(P=0.03)$ were all associated with an increased risk of CDI recurrence at 6 months.

\section{IBD Related Activity Over Time}

The impact of FMT on IBD-related activity at 2 and 6 months was a secondary endpoint. Overall, 6 out of 37 $(16.2 \%)$ and 15 out of $27(55.6 \%)$ subjects with follow-up at 2 and 6 months post FMT, respectively, had an IBD flare. A linear mixed-effects model was used to calculate the leastsquares means (LSM) of the HBI and Partial Mayo score over time. There was not a significant change in HBI scores over time $(P=0.84)$ when comparing baseline (LSM $6.6 ; 95 \% \mathrm{CI}$,

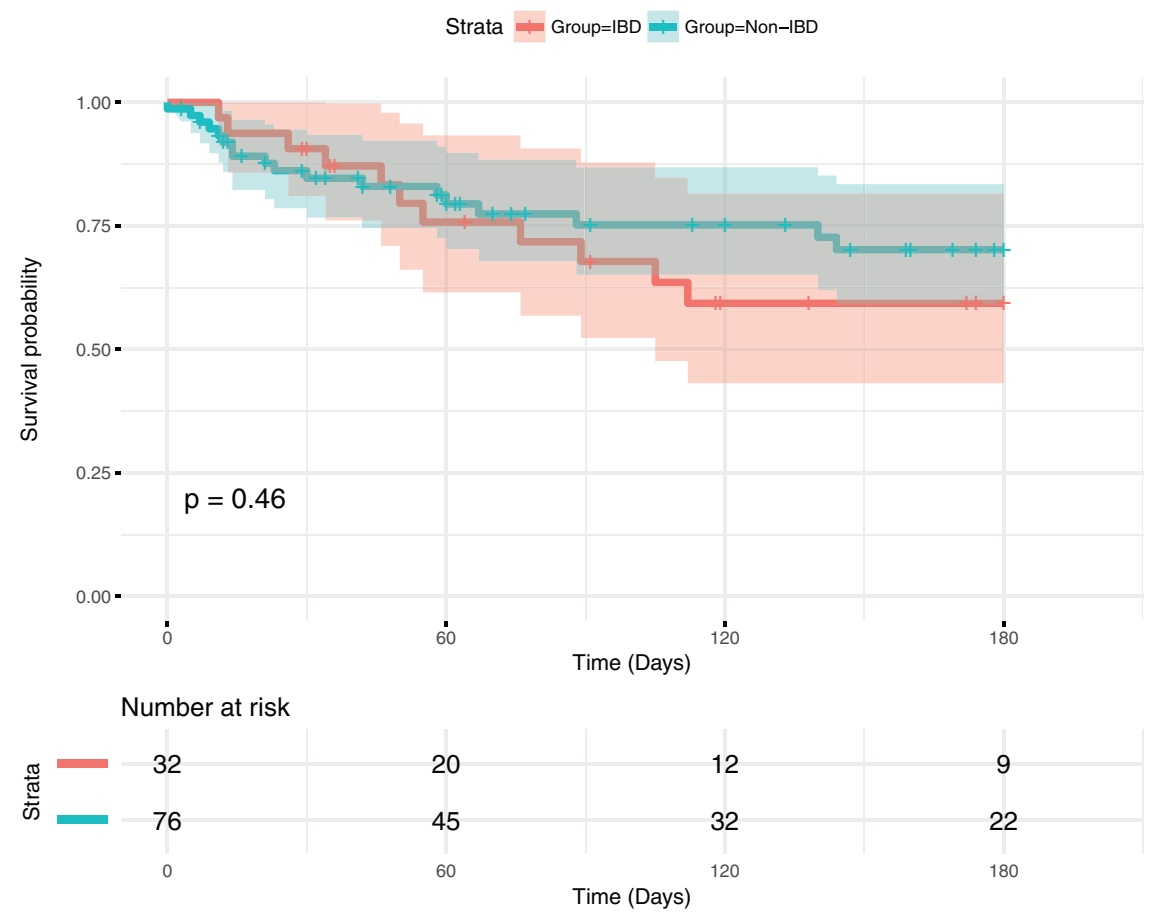

FIGURE 1. Survival analysis for time to first CDI recurrence from the date of initial FMT with log-rank test results. Censored at 6 months. 
TABLE 2. Univariate Analysis for Clostridium Difficile Recurrence at 2 and 6 Months

\begin{tabular}{|c|c|c|c|c|c|c|}
\hline & $\begin{array}{c}\text { CDIR } \\
2 \text { months }(\%)\end{array}$ & $\begin{array}{c}\text { No CDIR } \\
2 \text { months }(\%)\end{array}$ & P-value & $\begin{array}{c}\text { CDIR } \\
6 \text { months }(\%)\end{array}$ & $\begin{array}{c}\text { No CDIR } \\
6 \text { months (\%) }\end{array}$ & $P$ \\
\hline Hospitalization 90 days before FMT & $12(20)$ & $48(80)$ & 0.89 & $18(48.6 \%)$ & $19(51.4 \%)$ & 0.06 \\
\hline Age & $52 \pm 23.1$ & $53.5 \pm 22.3$ & 0.79 & $51.2 \pm 22.4$ & $50.6 \pm 23.1$ & 0.92 \\
\hline Female Sex & $15(20)$ & $60(80)$ & 0.85 & $18(36)$ & $32(64)$ & 0.75 \\
\hline PPI Use & $12(26.1)$ & $34(73.9)$ & 0.16 & $18(54.5)$ & $15(45.1)$ & 0.01 \\
\hline Number of CDI & $3.7 \pm 1.9$ & $3.5 \pm 1.8$ & 0.60 & $3.7 \pm 1.9$ & $3.5 \pm 1.5$ & 0.62 \\
\hline FMT Indication & & & 0.89 & & & 0.31 \\
\hline Refractory & $7(18.9)$ & $30(81.1)$ & & $11(45.8)$ & $13(54.2)$ & \\
\hline Recurrent & $16(20)$ & $64(80)$ & & $20(33.9)$ & $39(66.1)$ & \\
\hline Donor Type & & & 0.86 & & & 0.85 \\
\hline Fresh & $11(18.6)$ & $48(81.4)$ & & $17(36.2)$ & $30(63.8)$ & \\
\hline Frozen & $11(20)$ & $44(80)$ & & $13(38.2)$ & $21(61.8)$ & \\
\hline Location of Transplant & & & 0.28 & & & 0.02 \\
\hline Inpatient & $7(26.9)$ & $19(73.1)$ & & $10(62.5)$ & $6(37.5)$ & \\
\hline Outpatient & $16(17.4)$ & $76(82.6)$ & & $21(31.3)$ & $46(68.7)$ & \\
\hline \multicolumn{7}{|l|}{ Laboratory Findings at FMT } \\
\hline $\mathrm{WBC}$, mean $\pm \mathrm{SD}$ & $11.2 \pm 5.7$ & $10.4 \pm 10.6$ & 0.68 & $11.2 \pm 7.0$ & $9.2 \pm 11.1$ & 0.37 \\
\hline Hemoglobin, mean $\pm \mathrm{SD}$ & $10.5 \pm 2.3$ & $11.6 \pm 1.8$ & 0.10 & $10.8 \pm 2.1$ & $12.0 \pm 1.7$ & 0.02 \\
\hline Albumin, mean $\pm \mathrm{SD}$ & $3.2 \pm 0.8$ & $3.5 \pm 0.9$ & 0.14 & $3.4 \pm 0.8$ & $3.7 \pm 0.7$ & 0.09 \\
\hline IBD Type & & & 0.71 & & & 0.13 \\
\hline $\mathrm{CD}$ & $3(17.6)$ & $14(82.4)$ & & $3(23.1)$ & $10(76.9)$ & \\
\hline $\mathrm{UC}$ & $6(26.1)$ & $17(73.9)$ & & $9(50)$ & $9(50)$ & \\
\hline IBD Severity & & & 0.94 & & & 0.85 \\
\hline Remission & $1(12.5)$ & $7(87.5)$ & & $1(20)$ & $4(80)$ & \\
\hline Mild & $2(28.6)$ & $5(71.4)$ & & $2(33.3)$ & $4(66.7)$ & \\
\hline Moderate & $4(22.2)$ & $14(77.8)$ & & $6(40)$ & $9(60)$ & \\
\hline Severe & $1(16.7)$ & $5(83.3)$ & & $2(50)$ & $2(50)$ & \\
\hline IBD Severity & & & $>0.99$ & & & 0.63 \\
\hline Remission & $1(12.5)$ & $7(87.5)$ & & $1(20)$ & $4(80)$ & \\
\hline Active Disease & $7(22.6)$ & $24(77.4)$ & & $10(40)$ & $15(60)$ & \\
\hline Immunosuppression at FMT & $8(21.6)$ & $29(78.4)$ & 0.55 & $11(37.9)$ & $18(62.1)$ & $>0.99$ \\
\hline \multicolumn{7}{|l|}{ IBD Medications } \\
\hline Mesalamine & $3(23.1)$ & $10(76.9)$ & $>0.99$ & $4(44.4)$ & $5(55.6)$ & 0.70 \\
\hline AZA/MP/MTX & $0(0)$ & $7(100)$ & 0.18 & $0(0)$ & $6(100)$ & 0.06 \\
\hline Steroids & $2(13.3)$ & $13(86.7)$ & 0.44 & $4(36.4)$ & $7(63.6)$ & $>0.99$ \\
\hline Anti-TNF & $4(21.1)$ & $15(78.9)$ & $>0.99$ & $5(31.2)$ & $11(68.8)$ & 0.38 \\
\hline Vedolizumab & $1(14.3)$ & $6(85.7)$ & $>0.99$ & $2(33.3)$ & $4(66.7)$ & $>0.99$ \\
\hline
\end{tabular}

CDIR, clostridium difficile infection relapse; FMT, fecal microbiota transplant; PPI, proton pump inhibitor; CDI, clostridium difficile infection; WBC, white blood cell; SD, standard deviation; AZA, azathioprine; MP, mercaptopurine; MTX, methotrexate; Anti-TNF, anti-tumor necrosis factor.

4.3-8.9), 2-month (LSM 6.8; 95\% CI, 4.2-9.4), and 6-month (LSM 6.1; 95\% CI, 3.4-8.8) scores. Partial Mayo scores did not significantly change over time $(P=0.18)$ between baseline (LSM 3.9; 95\% CI, 3.1-4.6), 2-month (LSM 3.2; 95\% CI, 2.34.1) and 6-month (LSM 3.2; 95\% CI, 2.2-4.1) values.

\section{Microbiome of CDI Patients Pre-FMT}

The characteristics of the subjects with and without IBD whose microbiome was analyzed before FMT and up to 12 months after microbiota transplantation are detailed in Supplementary Table S2. The microbiome of patients before FMT was significantly different than their donors, with lower alpha diversity (Fig. 2A; student $t$ test, $P<0.001$ ), distinct beta diversity (Fig. 2B; PERMANOVA, $P=0.02$ ), and depletion in Bacteroides, Lachnospiraceae, and Faecalibacterium $(P<0.05)$. There were no significant differences between patients with and without IBD pre-FMT in alpha $(P=0.31)$ or beta diversity $(P=0.45)$, although subjects who experienced CDI recurrence 

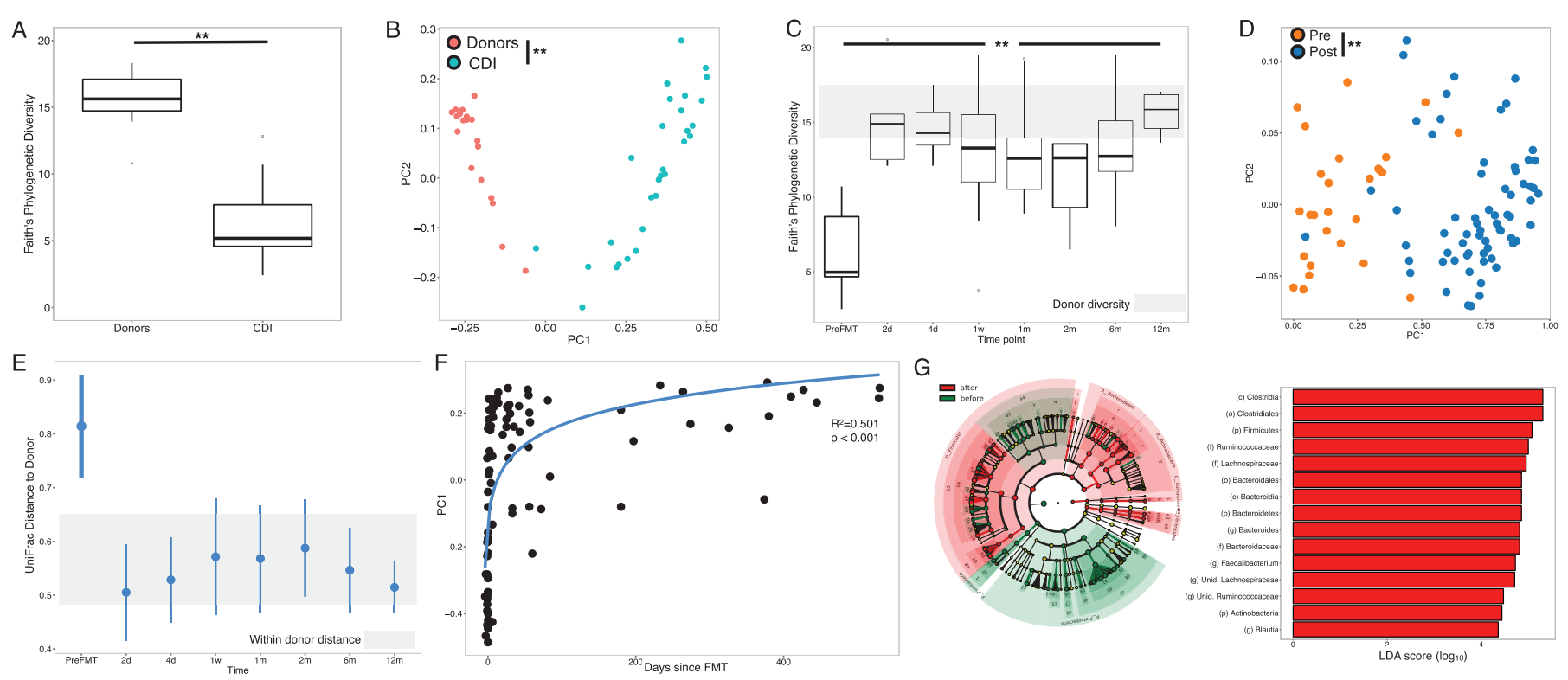

FIGURE 2. A, Alpha diversity of samples from donors $(n=19)$ and CDI patients $(n=29)$ pre-FMT $(P<0.05$, Student $t$ test). B, PCoA plot based on beta diversity distances of donors and CDI patients pre-FMT ( $p-0.02$, PERMANOVA). C, Boxplots indicating alpha diversity at each time point in all patient samples. Gray shaded area indicates the range of alpha diversity in donor samples. All time points had significantly higher diversity than pre-FMT, ANOVA with post hoc Tukey honestly significant difference (HSD) $(P<0.05)$. D, PCoA plot based on beta diversity distances before and after FMT $(P<0.05$, PERMANOVA). E, Engraftment (measured by UniFrac) distance from recipient to own donor in patients over time. Gray shaded area indicates distance within donors. At each time point, ranges indicate mean $+/$ - standard deviation of paired distances to donors. F, First principal coordinate from the PCoA plot vs time since transplant. Curve represents $\log _{10}$ fit $\left(R^{2}=0.501 ; P<0.05\right)$. G, LEfSe analysis comparing microbial composition before (green) and after (red) FMT. Represented are all taxa significantly distinct $(P<0.05)$ with LDA scores $>2.0$.

had significantly lower diversity than those who did not $(P=0.003)$.

\section{FMT Induces Significant Changes in Microbiome Composition and Diversity}

Alpha diversity increased significantly from $6.3 \pm 2.4$ before transplant to $13.4 \pm 3.5$ immediately after and remained high throughout the 12-month follow-up period (Fig. 2C; ANOVA $P<0.001$ ). Beta diversity was also significantly distinct before and after FMT (Fig. 2D; PERMANOVA, $P=0.001)$. Engraftment of donor microbiome into the patients was observed immediately after transplant and maintained for the duration of the study, as indicated by the decrease in microbiome UniFrac distances between recipient and donors post-FMT (Fig. 2E). Principal coordinate analysis $(\mathrm{PCoA})$ revealed a gradient along the first principal coordinate with time since transplant (Fig. $2 \mathrm{~F} ; \mathrm{R}^{2}=0.501$, $P=1.42 \mathrm{e}-15)$. These changes were mostly mediated by a significant enrichment in Bacteroides, Lachnospiraceae, Faecalibacterium, Blautia, and Ruminococcaceae after FMT (Fig. 2G). We further confirmed the replacement of the patients' microbiome using a random forest classifier, which could predict with high accuracy whether samples were obtained pre- or post-FMT based on microbiome composition alone (area under the curve: 0.978).

\section{Bacterial Engraftment After FMT Is Associated With Changes in IBD Treatment}

Although FMT results in significant changes in the microbiome of patients, we did not observe significant differences between the alpha diversity of patients with IBD and without IBD (Non-IBD) post-FMT ( $P>0.05$, all time points). Stratification of the IBD group based on disease activity, comparing those with mild endoscopic disease $(n=2)$ against those with moderate to severe endoscopic disease $(\mathrm{n}=6)$, revealed no significant differences either $(P>0.05)$. However, IBD patients who required a change in IBD-related medication after FMT (hereafter, "IBD escalation") exhibited a blunted increase in bacterial diversity immediately after FMT compared with those who did not require change in medications post-FMT ("IBD stable") (Fig. 3A, $P<0.05)$. Beta diversity was also altered in the IBD escalation group with significant differences between this group and all others (Fig. 3B; $P<0.05$ ). These changes immediately after FMT persist over time: alpha diversity of the IBD escalation patients failed to reach levels observed in the donors, whereas the stable IBD group and non-IBD group were within diversity levels of healthy donors (Fig. 4A). Importantly, we did not observe a similar pattern in patients who experienced CDI recurrence; although alpha diversity was lower than that of those patients who did not experience recurrence, neither of these groups had significantly lower diversity than the donors post-FMT (Fig. 4B). 

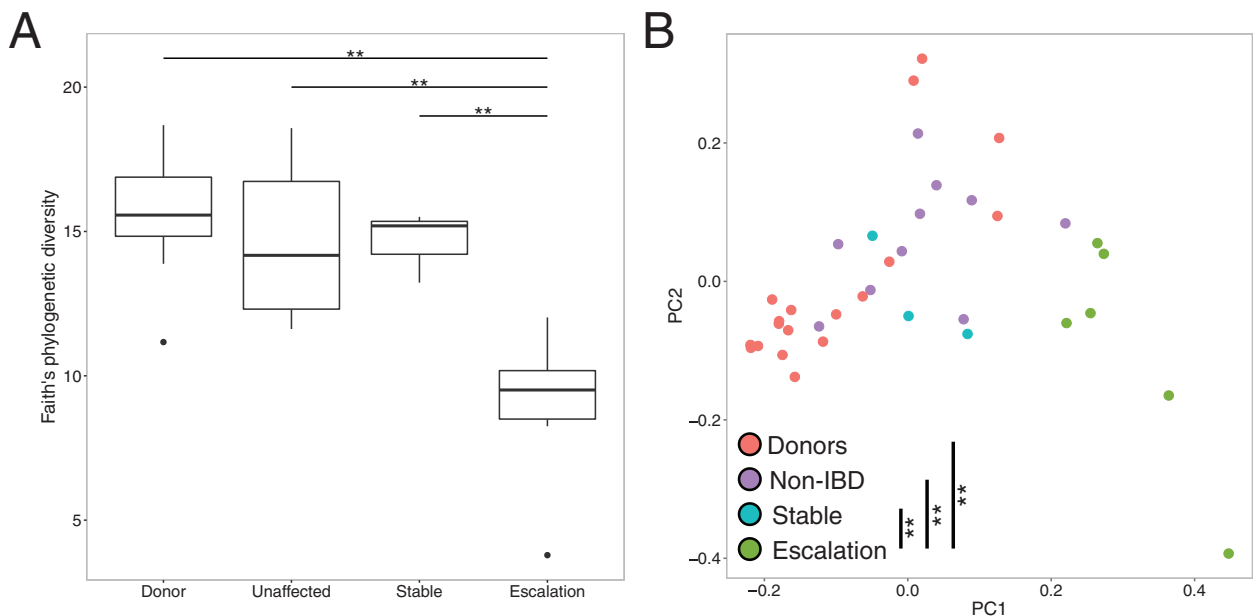

FIGURE 3. A, Alpha diversity in samples from donors $(n=19)$, non-IBD patients $(n=9)$, IBD escalation patients $(n=6)$, and IBD stable/remission patients $(n=3)$ immediately after FMT $(P<0.05$, ANOVA with post hoc Tukey HSD). B, PCoA plot based on UniFrac beta diversity distances from donors and patients immediately after FMT $(P<0.05$, PERMANOVA).
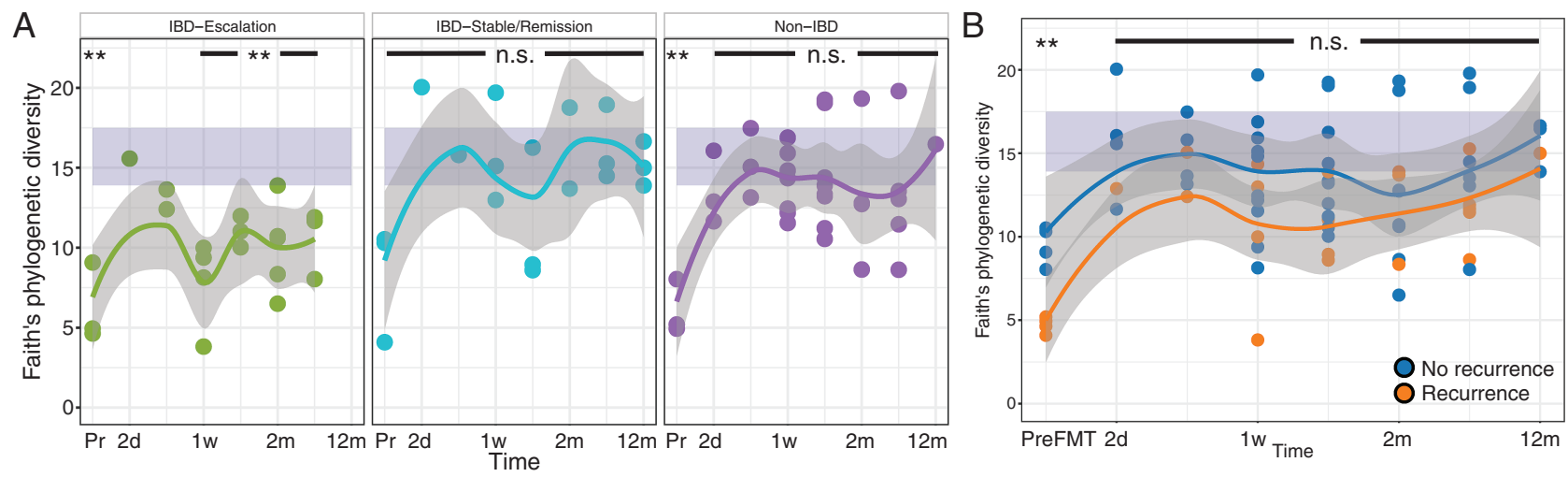

FIGURE 4. A, Alpha diversity in IBD escalation vs IBD stable/remission and non-IBD group immediately after FMT. B, Alpha diversity in patients with recurrence and non-recurrence. Curves smoothed over time using local regression, with gray area representing the confidence interval. Purple shaded area represents the diversity range in donors.

\section{IBD Therapy Escalation Is Associated With Functional Shifts in the Microbiome}

Bacterial functions were also significantly different between non-IBD/IBD stable and IBD escalation groups. We observed a decrease in pathways associated with nonsulfur-containing amino acids (lysine biosynthesis, histidine metabolism), enrichment in bacterial homeostasis during oxidative stress (glutathione metabolism), and enrichment in clinical disease activity (LPS biosynthesis) (Fig. 5). Overall, these results suggest an enrichment of functions associated with pathogenicity in the IBD escalation group.

\section{DISCUSSION}

We report the first study combining a long-term evaluation of the microbiome and the risk of recurrent CDI up to 6 months after FMT in a cohort of subjects with and without
IBD. Our results support 2 important conclusions: first, IBD does not significantly increase the risk of recurrent CDI after FMT; second, microbiome engraftment after fecal transplant is not influenced by the presence or absence of underlying IBD or the degree of disease activity but rather is impaired in subjects requiring escalation of IBD therapy.

Despite the extensive use of FMT, the mechanism underlying its success remains unclear, as evidenced by a recent publication demonstrating the efficacy of sterile filtrates in treating CDI. ${ }^{20}$ The vast majority of FMT studies to date have focused on efficacy outcomes at 1 to 3 months post-FMT, ${ }^{6,7}$ with few long-term studies published in the literature. ${ }^{21,22}$ Traditionally, recurrence rates are evaluated within 8 weeks of FMT; however, it is clinically important to understand the risk of CDI occurring outside of this period, prompting our evaluation of this long-term risk. Many studies exclude subjects with severe CDI, a known predictor of CDI recurrence, which may explain 

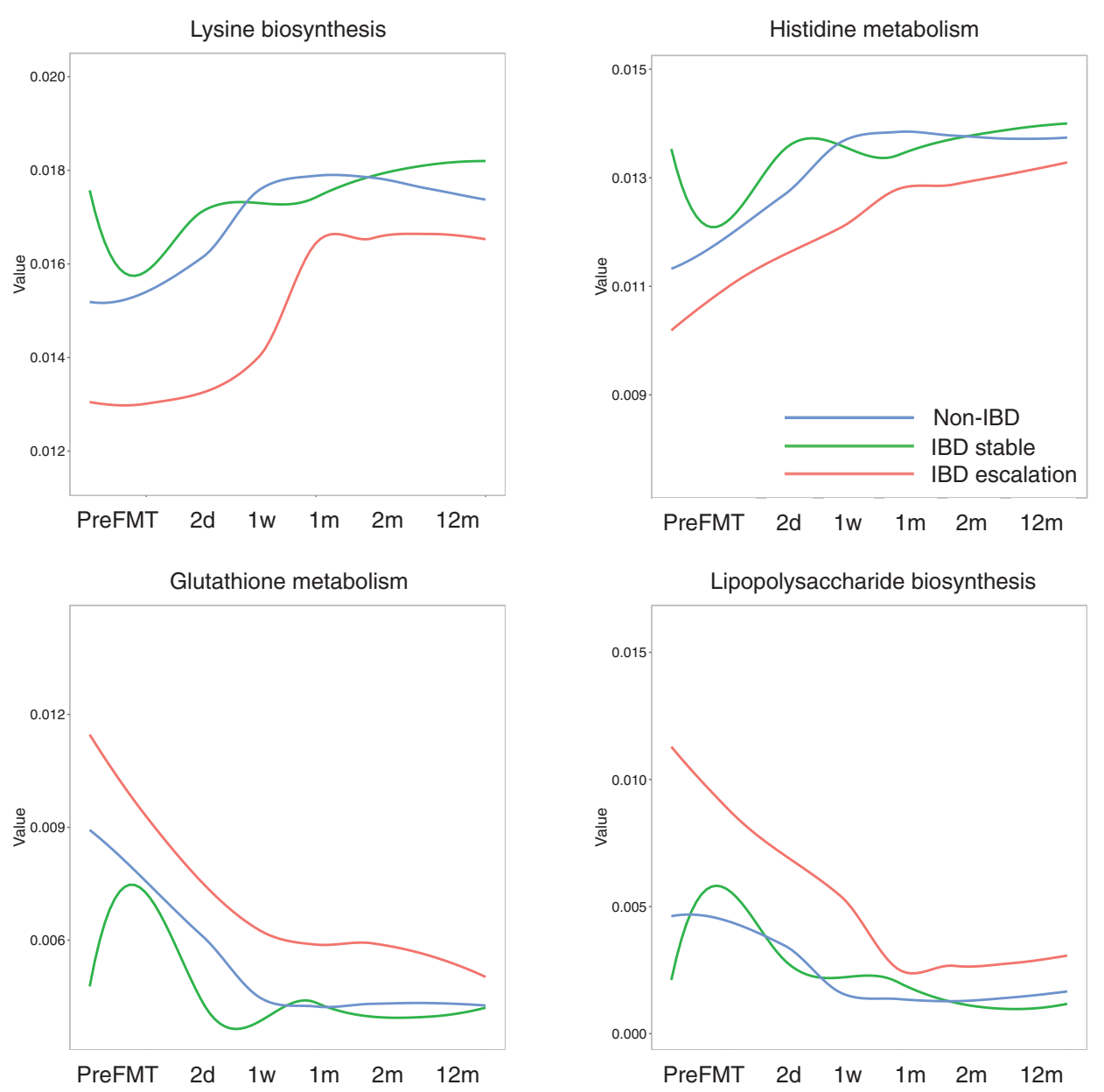

FIGURE 5. PICRUSt-predicted bacterial pathways differentially enriched in IBD escalation (red) vs IBD stable/remission (green) and non-IBD (blue). Curves smoothed over time using LOESS local regression.

the lower success rate of FMT observed in our cohort compared with others. ${ }^{6}$ However, our results indicate that there is a nontrivial late recurrence that occurs between 2 and 6 months (18.5\% vs $37.3 \%$ recurrence) post-FMT. Underlying IBD has been proposed as a risk factor for late recurrence of CDI. ${ }^{23}$ But in our cohort, we found no difference in recurrent CDI at 6 months between IBD and non-IBD patients. Identified risk factors for CDI recurrence after FMT have included severe $\mathrm{CDI}$, inpatient status, the number of previous CDIs, and a low albumin at the time of FMT. ${ }^{7,24}$ These predictors were for short-term relapse, generally within 2 months of fecal transplant. We identified long-term predictors of relapse including severe CDI, proton pump inhibitor use, and the comorbid condition hypertension. Low albumin and an inpatient location of FMT were not found to be predictive of FMT failure in our study; however, these reflect CDI severity, which we found to be predictive of recurrence.

There is continued controversy regarding the impact of IBD on the efficacy of FMT. Khoruts et al demonstrated a negative effect of IBD on the success of FMT, with 2-month CDI clearance rates of $74 \%$ vs $92.1 \%$ in those with and without IBD, whereas Fischer et al failed to identify IBD as a predictor of early failure. ${ }^{6,7}$ To address these discrepancies, our primary outcome was long-term CDI recurrence. At 2 and 6 months postFMT, underlying IBD was not found to influence recurrence and was not found to be a predictor of relapse. Additionally, we did not find IBD type or severity to be predictive of recurrence.

It has been hypothesized that a deficient immune response in subjects with IBD impacts the microbiome, explaining the reduced efficacy of FMT in subjects with IBD that has been observed in some studies. ${ }^{6}, 25,26$ Additionally, the presumed difference in efficacy may be impacted by ongoing symptoms in subjects with IBD secondary to disease activity, resulting in these patients being labeled as treatment failures. We longitudinally analyzed the microbiome in a subset of our patients to assess the impact of IBD on engraftment and its change over time. Although others have described a blunted increase in diversity in patients with concomitant IBD compared with those without, ${ }^{27}$ we observed no significant differences either before or after FMT. These findings support the clinical outcomes we 
observed, underscoring that IBD status does not necessarily impact the efficacy of FMT.

There is concern that the use of FMT to treat CDI can provoke a flare or worsening of underlying IBD activity. A recent meta-analysis found that the risk of an IBD flare after FMT is as high as $22.7 \%{ }^{8}$ We found that $16 \%$ of subjects developed a flare of their IBD within 2 months of their FMT, which is in line with previous reports from another large series. ${ }^{9}$ When examining the $\mathrm{HBI}$ and partial Mayo scores of the larger IBD cohort, we found no significant increase in IBD activity at 2 and 6 months post-FMT, showing that most patients tolerate FMT without an appreciable worsening of disease activity. This further reflects that those subjects with a flare post-FMT are generally able to have their disease brought under control. Although providers should be aware that the risk of disease flare after FMT exists, our findings support a relatively stable disease course over time.

Most previous studies have consistently found a rapid change in bacterial composition and diversity after transplantation, which is in concordance with our own results. However, it has not been well described whether CDI recurrence post-FMT is also associated with microbiome changes., ${ }^{58-30}$ Our analysis did not reveal any difference in diversity between subjects who did and did not recur after FMT. Subgroup analysis of the microbiome did not find differences in microbial diversity postFMT based on underlying IBD activity. These findings support our clinical finding that disease activity was not associated with an increased risk of CDI recurrence. Interestingly, when the microbiome of those with active IBD was analyzed, we noted a blunted increase in bacterial diversity in those that required medication escalation. Our result suggests that the changes in the post-FMT microbiome of CDI patients with IBD reported by others ${ }^{27}$ might in fact be associated to changes in therapy rather than to IBD itself. This hypothesis is supported by a recent report of numerous nonantibiotic drugs having a significant impact in the microbiome,,$^{31}$ although larger studies will be required to confirm our findings in CDI patients with IBD and delineate whether this blunted response may reflect another aspect of their disease state or infection impacting engraftment.

Our findings represent a single-center experience, and the retrospective nature of the study design is a limiting factor in data collection. Our center serves as a referral center, limiting the follow-up available for some patients and outcomes included in our analysis. Also, our definition of IBD flare relied on the determination of the treating physician. Furthermore, the number of patients in our microbiome analysis with active disease that required escalation is relatively small, although the differences in microbiome engraftment were significant after FMT and over time. The strengths of our study include the large number of subjects with and without IBD, allowing comparisons to be drawn between the 2 groups. Additionally, our cohort includes subjects with complex IBD and severe CDI, which are often excluded from other studies. The 6-month follow-up period is also an important strength, as it provides a longer assessment of FMT efficacy relative to many studies. ${ }^{6,7}$ Lastly, the longitudinal microbiome analysis in a subset of our patients provides important results regarding microbial engraftment over time in relation to IBD activity and therapy.

\section{CONCLUSION}

In conclusion, our study shows FMT to be a successful treatment of recurrent or severe CDI. Importantly, we did not find a difference in outcomes in subjects with or without IBD, supporting the hypothesis that underlying IBD does not decrease the efficacy of FMT. Microbiome analysis confirmed this observation, finding no significant differences between subjects with and without IBD nor between those who recur and those who do not. However, microbial engraftment was affected in those requiring escalation of IBD therapy, suggesting this is an important variable that should be accounted for in future studies.

\section{SUPPLEMENTARY DATA}

Supplementary data is available at Inflammatory Bowel Diseases online.

\section{REFERENCES}

1. Cohen SH, Gerding DN, Johnson S, et al.; Society for Healthcare Epidemiology of America; Infectious Diseases Society of America. Clinical practice guidelines for Clostridium difficile infection in adults: 2010 update by the Society for Healthcare Epidemiology of America (SHEA) and the Infectious Diseases Society of America (IDSA). Infect Control Hosp Epidemiol. 2010:31:431-455.

2. McFarland LV, Surawicz CM, Rubin M, et al. Recurrent clostridium difficile disease: epidemiology and clinical characteristics. Infect Control Hosp Epidemiol. 1999;20:43-50.

3. Surawicz CM, Brandt LJ, Binion DG, et al. Guidelines for diagnosis, treatment, and prevention of Clostridium difficile infections. Am J Gastroenterol. 2013;108:478-498; quiz 499.

4. Razik R, Rumman A, Bahreini Z, et al. Recurrence of Clostridium difficile infection in patients with inflammatory bowel disease: the RECIDIVISM study. $A m J$ Gastroenterol. 2016;111:1141-1146.

5. Seekatz AM, Aas J, Gessert CE, et al. Recovery of the gut microbiome following fecal microbiota transplantation. Mbio. 2014;5:e00893-e00814.

6. Khoruts A, Rank KM, Newman KM, et al. Inflammatory bowel disease affects the outcome of fecal microbiota transplantation for recurrent Clostridium difficile infection. Clin Gastroenterol Hepatol. 2016;14:1433-1438.

7. Fischer M, Kao D, Kelly C, et al. Fecal microbiota transplantation is safe and efficacious for recurrent or refractory Clostridium difficile infection in patient with inflammatory bowel disease. Inflamm Bowel Dis. 2016;22:2402-2409.

8. Qazi T, Amaratunga T, Barnes EL, et al. The risk of inflammatory bowel disease flares after fecal microbiota transplantation: systematic review and meta-analysis Gut Microbes. 2017;8:574-588.

9. Kelly CR, Ihunnah C, Fischer M, et al. Fecal microbiota transplant for treatment of Clostridium difficile infection in immunocompromised patients. Am J Gastroenterol. 2014;109:1065-1071.

10. Clemente JC, Pehrsson EC, Blaser MJ, et al. The microbiome of uncontacted amerindians. Sci Adv. 2015;1:pii e1500183.

11. Faith JJ, Guruge JL, Charbonneau M, et al. The long-term stability of the human gut microbiota. Science. 2013;341:1237439.

12. Magoč T, Salzberg SL. FLASH: fast length adjustment of short reads to improve genome assemblies. Bioinformatics. 2011;27:2957-2963.

13. Edgar RC. Search and clustering orders of magnitude faster than BLAST. Bioinformatics. 2010;26:2460-2461.

14. McDonald D, Price MN, Goodrich J, et al. An improved greengenes taxonomy with explicit ranks for ecological and evolutionary analyses of bacteria and archaea. Isme J. 2012;6:610-618.

15. Faith DP, Baker AM. Phylogenetic diversity (PD) and biodiversity conservation some bioinformatics challenges. Evol Bioinform Online. 2007;2:121-128. 
16. Lozupone C, Knight R. Unifrac: a new phylogenetic method for comparing microbial communities. Appl Environ Microbiol. 2005;71:8228-8235.

17. Caporaso JG, Kuczynski J, Stombaugh J, et al. QIIME allows analysis of high-throughput community sequencing data. Nat Methods. 2010;7:335-336.

18. Langille MG, Zaneveld J, Caporaso JG, et al. Predictive functional profiling of microbial communities using 16S rRNA marker gene sequences. Nat Biotechnol. 2013;31:814-821.

19. Parks DH, Tyson GW, Hugenholtz P, et al. STAMP: statistical analysis of taxonomic and functional profiles. Bioinformatics. 2014;30:3123-3124.

20. Ott SJ, Waetzig GH, Rehman A, et al. Efficacy of sterile fecal filtrate transfer for treating patients with Clostridium difficile infection. Gastroenterology. 2017;152:799-811.e7.

21. Brandt LJ, Aroniadis OC, Mellow M, et al. Long-term follow-up of colonoscopic fecal microbiota transplant for recurrent Clostridium difficile infection. Am J Gastroenterol. 2012;107:1079-1087.

22. Mamo Y, Woodworth MH, Wang T, et al. Durability and long-term clinical outcomes of fecal microbiota transplant treatment in patients with recurrent Clostridium difficile infection. Clin Infect Dis. 2018;66:1705-1711.

23. Newman KM, Rank KM, Vaughn BP, et al. Treatment of recurrent Clostridium difficile infection using fecal microbiota transplantation in patients with inflammatory bowel disease. Gut Microbes. 2017;8:303-309.
24. Fischer M, Kao D, Mehta SR, et al. Predictors of early failure after fecal microbiota transplantation for the therapy of Clostridium difficile infection: a multicenter study. Am J Gastroenterol. 2016;111:1024-1031.

25. Giesemann T, Guttenberg G, Aktories K. Human alpha-defensins inhibit Clostridium difficile toxin B. Gastroenterology. 2008;134:2049-2058.

26. Wehkamp J, Salzman NH, Porter E, et al. Reduced paneth cell alpha-defensins in ileal Crohn's disease. Proc Natl Acad Sci U S A. 2005;102:18129-18134.

27. Khanna S, Vazquez-Baeza Y, González A, et al. Changes in microbial ecology after fecal microbiota transplantation for recurrent $C$. Difficile infection affected by underlying inflammatory bowel disease. Microbiome. 2017;5:55.

28. Kelly CR, Khoruts A, Staley C, et al. Effect of fecal microbiota transplantation on recurrence in multiply recurrent Clostridium difficile infection: a randomized trial. Ann Intern Med. 2016;165:609-616.

29. Staley C, Kelly CR, Brandt LJ, et al. Complete microbiota engraftment is no essential for recovery from recurrent Clostridium difficile infection following fecal microbiota transplantation. MBio. 2016;7:e01965-16.

30. Hota SS, Sales V, Tomlinson G, et al. Oral vancomycin followed by fecal transplantation versus tapering oral vancomycin treatment for recurrent Clostridium difficile infection: an open-label, randomized controlled trial. Clin Infect Dis. 2017;64:265-271.

31. Maier L, Pruteanu M, Kuhn M, et al. Extensive impact of non-antibiotic drugs on human gut bacteria. Nature. 2018;555:623-628. 